\title{
SPECTRAL THEORY OF EXCEPTIONAL HERMITE POLYNOMIALS
}

\author{
DAVID GÓMEZ-ULLATE, YVES GRANDATI, AND ROBERT MILSON
}

\begin{abstract}
In this paper we revisit exceptional Hermite polynomials from the point of view of spectral theory, following the work initiated by Lance Littlejohn. Adapting a result of Deift, we provide an alternative proof of the completeness of these polynomial families. In addition, using equivalence of Hermite Wronskians we characterize the possible gap sets for the class of exceptional Hermite polynomials.
\end{abstract}

\section{INTRODUCTION}

Consider a Sturm-Liouville problem (SLP) on $(-\infty, \infty)$ :

$$
-\left(W y^{\prime}\right)^{\prime}-R y=\varepsilon W y,
$$

where $W, R=O\left(e^{-x^{2}}\right)$ as $|x| \rightarrow \infty$. Remarkably, there exist a large class of such eigenvalue problems with polynomial eigenfunctions. Classical Hermite polynomials, which correspond to the case of $W=e^{-x^{2}}, R=0$, are just one, very particular, example. Families of polynomials that arise as eigenfunctions of such SLP are called exceptional Hermite orthogonal polynomials.

Being the eigenfunctions of an SLP, exceptional Hermite polynomials are complete families of orthogonal polynomials with weight $W(x)>0, x \in(-\infty, \infty)$ [10, 8]. However, unlike the classical Hermite polynomials, the degree sequence of an exceptional family has a finite number of gaps in the degree sequence; there is a finite number of so-called exceptional degrees for which there is no corresponding eigenpolynomial.

The well-known Bochner theorem [1] asserts that if $y_{n}(x), n=0,1,2, \ldots$ with $\operatorname{deg} y_{n}=n$ is a family of polynomials that satisfies a second-order eigenvalue equation

$$
p y_{n}^{\prime \prime}+q y_{n}^{\prime}+r y_{n}=\varepsilon_{n} y,
$$

then necessarily $p(x), q(x)$ are polynomials with $\operatorname{deg} p \leq 2, \operatorname{deg} q \leq 1$ and $r$ is a constant. Multiplying (1) by $-W^{-1}$ gives

$$
y^{\prime \prime}+q y^{\prime}+r y=-\varepsilon y
$$

where

$$
q=W^{-1} W^{\prime}, \quad r=W^{-1} R
$$

In order for (2) to have infinitely many polynomial eigenfunctions, it is necessary for $q(x), r(x)$ to be rational functions with $\operatorname{deg} q \leq 1$ and $\operatorname{deg} r \leq 0$. To obtain nonclassical polynomials, it is necessary for $q$ and $r$ to have poles. Bochner's theorem then implies that such a non-classical family of polynomial eigenfunctions must have gaps in the degree sequence. 
If we assume that there is at most a finite number of such gaps, then it is possible to show (see 6, 8]) that, necessarily, (2) takes the form

$$
y^{\prime \prime}-2\left(x+\frac{\eta^{\prime}}{\eta}\right) y^{\prime}+\left(\frac{\eta^{\prime \prime}}{\eta}+2 x \frac{\eta^{\prime}}{\eta}\right) y=\varepsilon y,
$$

where $\eta(x)$ is a real polynomial without any real zeros . Not every choice of $\eta$ results in an eigenvalue relation (3) with polynomial eigenfunctions. When it does, however, one can show that there are precisely $\operatorname{deg} \eta$ "exceptional" degrees.

Some examples of exceptional polynomials were investigated back in the early $90 \mathrm{~s}$ 4 but their systematic study started about 10 years ago, where a full classification was given for codimension one [10. The role of Darboux transformations in the construction process was quickly recognized [11, 25, 28, and the next conceptual step involved the generation of exceptional families by multiple-step or higher order Darboux transformations 12 .

Exceptional polynomials appear in mathematical physics as bound states of exactly solvable rational extensions [8, 23, 25] and exact solutions to Dirac's equation 26]. They appear also in connection with super-integrable systems [24, 22] and finite-gap potentials [16]. From a mathematical point of view, the main results are concerned with the full classification of exceptional polynomials 6], properties of their zeros [14, 17, 18, and recurrence relations [5, 13, 19].

Lance Littlejohn and collaborators wrote a series of papers analyzing the spectraltheoretic properties of 1-step exceptional operators [20, 21. It is the ambition of the present work to extend this type of analysis to the class of multi-step exceptional Hermite operators. There are two primary results. First, we merge the approach of Littlejohn with spectral-theoretic characterization of Darboux transformations obtained by Deift [3. (see 15] for a further extension to the double-commutator method) to provide a novel demonstration of the completeness of the exceptional polynomials. Second, we characterize the possible gap sets of families of exceptional Hermite polynomial in terms the corresponding partition.

\section{SOME SPECTRAL THEORY}

Let $\mathcal{R} \subset \mathbb{R}[x]$ be the set of real-valued polynomials that have no real zeros. Consider a SLP on $(-\infty, \infty)$ of the form

$$
-\left(W_{\eta} y^{\prime}\right)^{\prime}-R_{\eta} y=\varepsilon W_{\eta} y, \quad \eta \in \mathcal{R},
$$

where

$$
\begin{aligned}
W_{\eta} & :=\eta^{-2} e^{-x^{2}} \\
R_{\eta} & :=\eta^{-3}\left(\eta^{\prime \prime}+2 x \eta^{\prime}\right) e^{-x^{2}}
\end{aligned}
$$

In this section we will consider Darboux transformations of self-adjoint operators corresponding to SLP belonging to the class shown in (4).

Fix $\xi, \eta \in \mathcal{R}$ and set

$$
\begin{aligned}
& \tilde{\eta}:=\eta e^{x^{2}} \\
& \hat{\eta}:=\eta^{-1} e^{-\frac{x^{2}}{2}},
\end{aligned}
$$

with $\tilde{\xi}, \hat{\xi}$ defined analogously. 
Next, introduce the bilinear differential expression

$$
\chi(f, g)=f g^{\prime \prime}-2 f^{\prime} g^{\prime}+f^{\prime \prime} g-2 x\left(f g^{\prime}-f^{\prime} g\right) .
$$

and define the following first, and second order differential expressions

$$
\begin{aligned}
\alpha_{\xi, \eta} y & =\eta^{-1} \operatorname{Wr}(\xi, y) \\
\beta_{\eta, \xi} & =\alpha_{\tilde{\eta}, \tilde{\xi}} \\
\tau_{\eta} y & =\eta^{-1} \chi(\eta, y) .
\end{aligned}
$$

with $\tau_{\xi}$ defined analogously. Note that $\tau_{\eta}$ is precisely the second-order differential expression in the left-side of (3).

A direct calculation shows that

$$
\left(\alpha_{\xi, \eta} f\right) g W_{\xi}+f\left(\beta_{\eta, \xi} g\right) W_{\eta}=(\hat{\eta} f \hat{\xi} g)^{\prime},
$$

with $W_{\eta}, W_{\xi}$ as defined in (5). Consequently, $\alpha_{\xi, \eta}$ and $-\beta_{\eta, \xi}$ are formally adjoint with respect to weights $W_{\xi}, W_{\eta}$. To be precise, if $I \subset \mathbb{R}$ is a compact interval, and $f, g$ sufficiently smooth functions defined on $I$, we have

$$
\int_{I}\left(\alpha_{\xi, \eta} f\right) \bar{g} W_{\xi}+\int_{I} f\left(\beta_{\eta, \xi} \bar{g}\right), W_{\eta}=\left.\hat{\eta} f \hat{\xi} \bar{g}\right|_{I}
$$

Let us also recall Lagrange's identity

$$
\left(\tau_{\eta} f\right) g W_{\eta}-f\left(\tau_{\eta} g\right) W_{\eta}=W_{\eta} \operatorname{Wr}(f, g) .
$$

Consequently, $\tau_{\eta}$ is symmetric with respect to $W_{\eta}$ in the sense that

$$
\int_{I}\left(\tau_{\eta} f\right) \bar{g} W_{\eta}-\int_{I} f\left(\tau_{\eta} \bar{g}\right) W_{\eta}=\left.W_{\eta} \operatorname{Wr}(f, \bar{g})\right|_{I},
$$

An analogous relation holds for $\tau_{\xi}$ and $W_{\xi}$.

Let $\left(\mathcal{H}_{\eta},\langle\cdot, \cdot\rangle_{\eta}\right)=\mathrm{L}^{2}\left(\mathbb{R}, W_{\eta}\right)$ be the Hilbert space of square-integrable complexvalued functions defined on $(-\infty, \infty)$, with $\mathcal{H}_{\xi}$ defined analogously. Let $\mathcal{D}_{0}$ denote the vector space of smooth, functions with compact support, and let

$$
\mathcal{D}_{\xi}:=\left\{f \in \mathcal{C}^{\infty}(\mathbb{R}): \operatorname{Wr}(f, \xi)(x) \equiv 0 \text { for }|x| \text { sufficiently large }\right\} .
$$

In other words, an element of $\mathcal{D}_{\xi}$ behaves like a multiple of $\xi(x)$ outside a compact interval, but with the constant of proportionality for large $x$ not necessarily equal to the constant of proportionality for small $x$. It is well known that $\mathcal{D}_{0}$ is dense in $\mathcal{H}_{\eta}$ and $\mathcal{H}_{\xi}$. Since elements of $\mathcal{D}_{\xi}$ are square integrable with respect to $W_{\eta}$ and since $\mathcal{D}_{0} \subset \mathcal{D}_{\xi}$, the latter is also a dense subspace.

Let $a_{\xi, \eta}: \mathcal{H}_{\eta} \rightarrow \mathcal{H}_{\xi}, b_{\eta, \xi}: \mathcal{H}_{\xi} \rightarrow \mathcal{H}_{\eta}$ denote densely defined first-order operators with action $\alpha_{\xi, \eta}, \beta_{\eta, \xi}$, respectively, and with

$$
\text { Dom } a_{\xi, \eta}=\mathcal{D}_{\xi}, \quad \operatorname{Dom} b_{\eta, \xi}=\mathcal{D}_{0} .
$$

Since $\alpha_{\xi, \eta} \xi \equiv 0$, it follows that $\operatorname{Ran} a_{\xi, \eta}=\mathcal{D}_{0}$. For same reason and by (14), we have $\operatorname{Ran} b_{\eta, \xi} \subset \operatorname{Ann}_{\eta} \xi$, where

$$
\operatorname{Ann}_{\eta} \xi=\left\{f \in \mathcal{H}_{\eta}:\langle f, \xi\rangle_{\eta}=0 .\right\} .
$$

Next, we strengthen these assertions as follows.

Proposition 2.1. We have $\overline{\operatorname{Ran} a_{\xi, \eta}}=\mathcal{H}_{\xi}$. 
Proof. Since $\mathcal{D}_{0}$ is dense in $\mathcal{H}_{\xi}$, it suffices to show that $\mathcal{D}_{0} \subset \operatorname{Ran} a_{\xi, \eta}$. Let $h \in \mathcal{D}_{0}$ be given. Set

$$
f(x)=\xi(x) \int_{-\infty}^{x} h \eta \xi^{-2}, \quad x \in \mathbb{R} .
$$

Since $h \in \mathcal{D}_{0}$, for $x$ sufficiently small, we have $f(x)=0$. For $x$ sufficiently large, we have $f(x)=C \xi(x)$ where $C=\int_{\mathbb{R}} h \eta \xi^{-2}$. Hence, $f \in \mathcal{D}_{\xi}$. By (10) we have,

$$
\alpha_{\xi, \eta} f=\eta^{-1} \xi^{2}\left(\xi^{-1} f\right)^{\prime}=h .
$$

Proposition 2.2. We have $\overline{\operatorname{Ran} b_{\eta, \xi}}=\mathrm{Ann}_{\eta} \xi$.

Proof. Let $\left\{f_{n} \in \mathcal{D}_{0}\right\}_{n \in \mathbb{N}}$ be a sequence such that $\beta_{\eta, \xi} f_{n} \stackrel{n}{\longrightarrow} h$ for some $h \in \mathcal{H}_{\eta}$. Since $\alpha_{\xi, \eta} \xi=0$, by (13), we have

$$
\left\langle\beta_{\eta, \xi} f_{n}, \xi\right\rangle_{\eta}=0, \quad n \in \mathbb{N} .
$$

Moreover, since

$$
\langle h, \xi\rangle_{\eta}=\left\langle h-\beta_{\eta, \xi} f_{n}, \xi\right\rangle_{\eta} \stackrel{n}{\longrightarrow} 0,
$$

we have $h \in \mathrm{Ann}_{\eta} \xi$. Hence, $\overline{\operatorname{Ran} b_{\eta, \xi}} \subseteq \mathrm{Ann}_{\eta} \xi$.

We now prove the converse. Let $h \in \mathrm{Ann}_{\eta} \xi$ be given. Let $\left\{h_{n} \in \mathcal{D}_{0}\right\}_{n \in \mathbb{N}}$ be a sequence such that $\left\|h_{n}-h\right\| \stackrel{n}{\longrightarrow} 0$. Choose a $p \in \mathcal{D}_{0}$ such that $\langle p, \xi\rangle \neq 0$, and set

$$
g_{n}=h_{n}-\frac{\left\langle h_{n}, \xi\right\rangle_{\eta}}{\langle p, \xi\rangle_{\eta}} p \in \mathcal{D}_{0}, \quad n \in \mathbb{N} .
$$

By construction, $g_{n} \in \operatorname{Ann}_{\eta} \xi, n \in \mathbb{N}$. By assumption, $\left\langle h_{n}, \xi\right\rangle_{\eta} \stackrel{n}{\longrightarrow} 0$. Hence,

$$
\left\|g_{n}-h\right\|_{\eta} \leq\left\|h_{n}-h\right\|_{\eta}+\left|\frac{\left\langle h_{n}, \xi\right\rangle_{\eta}}{\langle p, \xi\rangle_{\eta}}\right|\|p\|_{\eta} \stackrel{n}{\longrightarrow} 0 .
$$

For $n \in \mathbb{N}$, set

$$
f_{n}(x)=\tilde{\eta}(x) \int_{-\infty}^{x} g_{n} \tilde{\xi} \tilde{\eta}^{-2}=\tilde{\eta}(x) \int_{-\infty}^{x} g_{n} \xi W_{\eta}, \quad x \in \mathbb{R} .
$$

Since $g_{n}$ has compact support, $f_{n}(x)=0$ for $x$ sufficiently small. Since $g_{n} \in \mathrm{Ann}_{\eta} \xi$, we also have $f_{n}(x)=0$ for $x$ sufficiently large. Hence, $f_{n} \in \mathcal{D}_{0}$. Observe that

$$
\beta_{\eta, \xi} f_{n}=\tilde{\xi}^{-1} \tilde{\eta}^{2}\left(\tilde{\eta}^{-1} f_{n}\right)^{\prime}=g_{n} .
$$

Hence $g_{n} \in \operatorname{Ran} b_{\eta, \xi}, n \in \mathbb{N}$. We already showed that $g_{n} \stackrel{n}{\longrightarrow} h$. This proves that

$$
\operatorname{Ann}_{\eta} \xi \subseteq \overline{\operatorname{Ran} b_{\eta, \xi}} .
$$

Let $A_{\xi, \eta}, B_{\eta, \xi}$ denote the maximal extensions of $a_{\xi, \eta}, b_{\eta, \xi}$, respectively. Formally,

$$
\begin{aligned}
& \operatorname{Dom} A_{\xi, \eta}=\left\{f \in \mathcal{H}_{\eta}: f \in \mathrm{AC}_{\mathrm{loc}}(\mathbb{R}) ; \alpha_{\xi, \eta} f \in \mathcal{H}_{\xi}\right\}, \\
& \operatorname{Dom} B_{\eta, \xi}=\left\{g \in \mathcal{H}_{\xi}: g \in \mathrm{AC}_{\mathrm{loc}}(\mathbb{R}) ; \beta_{\eta, \xi} g \in \mathcal{H}_{\eta}\right\} .
\end{aligned}
$$

Proposition 2.3. For $f \in \operatorname{Dom} A_{\xi, \eta}$ and $g \in \operatorname{Dom} B_{\eta, \xi}$, we have

$$
\left\langle A_{\xi, \eta} f, g\right\rangle_{\xi}=\left\langle f, B_{\eta, \xi} g\right\rangle_{\eta} .
$$


Proof. By (14), we have

$$
\left.\hat{\eta} f \hat{\xi} g\right|_{-t} ^{t} \rightarrow\left\langle\alpha_{\xi, \eta} f, g\right\rangle_{\eta}-\left\langle f, \beta_{\eta, \xi} g\right\rangle_{\xi} \quad \text { as } t \rightarrow \infty
$$

with $\hat{\eta}, \hat{\xi}$ as per (8). Observe that

$$
(\hat{\eta} f)^{2}=f^{2} W_{\eta}, \quad(\hat{\xi} g)^{2}=g^{2} W_{\xi} .
$$

Hence, $\hat{\eta} f, \hat{\xi} g \in \mathrm{L}^{2}(\mathbb{R})$. Therefore, the above limit must be zero.

Proposition 2.4. We have $b_{\eta, \xi}^{*} \subseteq-A_{\xi, \eta}$ and $a_{\xi, \eta}^{*} \subseteq-B_{\eta, \xi}$.

Proof. Let $f \in \operatorname{Dom} b_{\eta, \xi}^{*} \subset \mathcal{H}_{\eta}$. By definition, there exists an $h \in \mathcal{H}_{\xi}$ such that

$$
\left\langle f, \beta_{\eta, \xi} g\right\rangle_{\eta}=\langle h, g\rangle_{\xi}, \quad \text { for all } g \in \mathcal{D}_{0} \text {. }
$$

Set

$$
\tilde{f}(x):=\xi \int_{0}^{x} h \eta \xi^{-2}, \quad x \in \mathbb{R} .
$$

Hence, by (10), we have $\alpha_{\xi, \eta}[\tilde{f}]=h$ almost everywhere. By (14), it then follows that

Hence,

$$
\int_{\mathbb{R}} \tilde{f}\left(\beta_{\eta, \xi} \bar{g}\right) W_{\eta}=\langle h, g\rangle_{\xi}, \quad \text { for all } g \in \mathcal{D}_{0} .
$$

$$
\int_{\mathbb{R}}(f-\tilde{f})\left(\beta_{\eta, \xi} \bar{g}\right) W_{\eta}=0 \text { for all } g \in \mathcal{D}_{0} .
$$

Now, $f-\tilde{f} \in \mathrm{L}^{2}\left(I, W_{\eta}\right)$ for every compact interval $I \subset \mathbb{R}$. Hence, by Proposition 2.2. $f$ and $\tilde{f}$ differ by a multiple of $\xi$ a.e. on $I$. Since this is true for every $I$, it follows that $f-\tilde{f}$ is a multiple of $\xi$ a.e. on all of $\mathbb{R}$. Therefore, $f \in \operatorname{Dom} A_{\xi, \eta}$ with $\alpha_{\xi, \eta} f=h$.

Next, let $f \in \operatorname{Dom} a_{\xi, \eta}^{*}$. By definition, there exists an $h \in \mathcal{H}_{\eta}$ such that

$$
\left\langle f, \alpha_{\xi, \eta} g\right\rangle_{\xi}=\langle h, g\rangle_{\eta}, \quad \text { for all } g \in \mathcal{D}_{\xi} \subset \mathcal{H}_{\eta} .
$$

If $g=\xi$, then the above expressions vanish. Hence, $h \in \mathrm{Ann}_{\eta} \xi$, which implies that

$$
\tilde{f}(x):=\tilde{\eta}(x) \int_{-\infty}^{x} h \xi W_{\eta}, \quad x \in \mathbb{R}
$$

is well defined. By construction, $\beta_{\eta, \xi} \tilde{f}=h$ a.e. It follows that

$$
\int_{\mathbb{R}} \tilde{f}\left(\alpha_{\xi, \eta} \bar{g}\right) W_{\xi}=\langle h, g\rangle_{\eta}, \quad \text { for all } g \in \mathcal{D}_{\xi}
$$

Hence,

$$
\int_{\mathbb{R}}(f-\tilde{f})\left(\alpha_{\xi, \eta} \bar{g}\right) W_{\xi}=0 \text { for all } g \in \mathcal{D}_{\xi} .
$$

Therefore, by Proposition 2.1 $f=\tilde{f}$ a.e. on $\mathbb{R}$.

Proposition 2.5. We have $A_{\xi, \eta}=-B_{\eta, \xi}^{*}$ and $-B_{\eta, \xi}=A_{\xi, \eta}^{*}$.

Proof. By Proposition 2.3, we have $A_{\xi, \eta} \subseteq-B_{\eta, \xi}^{*}$ and $-B_{\eta, \xi} \subseteq A_{\xi, \eta}^{*}$. By Proposition 2.4 .

$$
-B_{\eta, \xi}^{*} \subseteq-b_{\eta, \xi}^{*} \subseteq A_{\xi, \eta}, \quad \text { and } \quad A_{\xi, \eta}^{*} \subseteq a_{\xi, \eta}^{*} \subseteq-B_{\eta, \xi},
$$

as was to be shown. 
Let $T_{\eta}: \mathcal{H}_{\eta} \rightarrow \mathcal{H}_{\eta}$ denote the densely defined operator with action $\tau_{\eta}$ and with maximal domain

$$
\operatorname{Dom}\left(T_{\eta}\right)=\left\{f \in \mathrm{AC}_{\mathrm{loc}}^{1}(\mathbb{R}): \tau_{\eta} f \in \mathcal{H}_{\eta}\right\}
$$

Let $T_{\xi}: \mathcal{H}_{\xi} \rightarrow \mathcal{H}_{\xi}$ be the analogous operator with action $\tau_{\xi}$.

Proposition 2.6. Let $\eta, \xi \in \mathcal{R}$ be such that

$$
\chi(\eta, \xi)=\varepsilon_{0} \eta \xi, \quad \varepsilon_{0} \in \mathbb{R} .
$$

Then,

$$
\begin{aligned}
& T_{\eta}=B_{\eta, \xi} A_{\xi, \eta}+\varepsilon_{0}, \\
& T_{\xi}=A_{\xi, \eta} B_{\eta, \xi}+\varepsilon_{0}+2
\end{aligned}
$$

in the the sense of naturally defined composition; i.e.

$$
\begin{aligned}
& \operatorname{Dom} B_{\eta, \xi} A_{\xi, \eta}=\left\{f \in \operatorname{Dom} A_{\xi, \eta}: A_{\xi, \eta} f \in \operatorname{Dom} B_{\eta, \xi}\right\} ; \\
& \operatorname{Dom} A_{\xi, \eta} B_{\eta, \xi}=\left\{f \in \operatorname{Dom} B_{\eta, \xi}: B_{\eta, \xi} f \in \operatorname{Dom} A_{\xi, \eta}\right\} .
\end{aligned}
$$

Lemma 2.1. Relation (21) is equivalent to either of the following:

$$
\begin{aligned}
\tau_{\eta} \xi & =\varepsilon_{0} \xi, \\
\tau_{\xi} \tilde{\eta} & =\left(\varepsilon_{0}+2\right) \tilde{\eta} .
\end{aligned}
$$

Proof. The equivalence of (21) and (23) follows directly from the definition (12). The following differential relation can be verified by direct calculation:

$$
\chi(\xi, \tilde{\eta})=e^{x^{2}} \chi(\eta, \xi)+2 \xi \tilde{\eta}
$$

Hence,

$$
\tilde{\eta} \tau_{\eta} \xi=e^{x^{2}} \chi(\eta, \xi)=\chi(\xi, \tilde{\eta})-2 \xi \tilde{\eta}=\xi\left(\tau_{\xi}-2\right) \tilde{\eta}
$$

This proves the equivalence of (23) and (24).

Lemma 2.2. Suppose that (21) holds. Then,

$$
\begin{aligned}
\tau_{\eta} & =\beta_{\eta, \xi} \alpha_{\xi, \eta}+\varepsilon_{0}, \\
\tau_{\xi} & =\alpha_{\xi, \eta} \beta_{\eta, \xi}+\varepsilon_{0}+2
\end{aligned}
$$

Proof. By definition and direct calculation,

$$
\chi(\eta, f) \xi-\chi(\eta, \xi) f=\tilde{\eta}^{-1} \operatorname{Wr}(\eta \tilde{\eta}, \operatorname{Wr}(\xi, f))
$$

Hence, by (10) - (12),

$$
\begin{aligned}
\tau_{\eta} f & =\left(\tilde{\xi} \eta^{2}\right)^{-1} \operatorname{Wr}(\eta \tilde{\eta}, \operatorname{Wr}(\xi, f))+\xi^{-1} f \tau_{\eta} \xi \\
& =\tilde{\xi}^{-1} \operatorname{Wr}\left(\tilde{\eta}, \eta^{-1} \operatorname{Wr}(\xi, f)\right)+\varepsilon_{0} f \\
& =\beta_{\eta, \xi} \alpha_{\xi, \eta} f+\varepsilon_{0} f
\end{aligned}
$$

By definition and direct calculation,

$$
\begin{aligned}
\alpha_{\tilde{\eta}, \xi} f & =e^{x^{2}} \beta_{\eta, \xi} f, \\
\beta_{\xi, \tilde{\eta}} f & =e^{-2 x^{2}} \eta^{-1} \operatorname{Wr}\left(e^{x^{2}} \xi, f\right)=\eta^{-1} \operatorname{Wr}\left(\xi, e^{-x^{2}} f\right)=\alpha_{\xi, \eta}\left(e^{-x^{2}} f\right)
\end{aligned}
$$

Applying (26) with $\eta \mapsto \xi, \xi \mapsto \tilde{\eta}, \varepsilon_{0} \mapsto \varepsilon_{0}+2$, and using (24) gives

$$
\tau_{\xi}=\beta_{\xi, \tilde{\eta}} \alpha_{\tilde{\eta}, \xi}+\varepsilon_{0}+2=\alpha_{\xi, \eta} \beta_{\eta, \xi}+\varepsilon_{0}+2 \text {. }
$$


Proof. By Lemma 2.2 the formal factorization relations (26) and (27) hold. The differential expressions $\tau_{\eta}, \tau_{\xi}$ are limit point at $\pm \infty$; see [2, Theorem 2.4]. Hence, $T_{\eta}, T_{\xi}$ are self-adjoint. By Proposition 2.5. $A_{\xi, \eta},-B_{\eta, \xi}$ are adjoint. Hence, the natural compositions $A_{\xi, \eta} B_{\eta, \xi}$ and $B_{\eta, \xi} A_{\xi, \eta}$ are self-adjoint by a standard theorem in functional analysis; see for example [27, Theorem 13.13]. Since there are no boundary conditions at $\pm \infty$ the LHS and the RHS of (22) must coincide.

Proposition 2.7. Let $\eta, \xi \in \mathcal{R}$ such that (21) holds. Let $\psi \in \mathcal{H}_{\eta}$ be an eigenfunction of $T_{\eta}$; i.e. $\tau_{\eta} \psi=\varepsilon \psi, \varepsilon \in \mathbb{R}$. Then, necessarily $\varepsilon_{0}>\varepsilon$ and

$$
\hat{\psi}:=\alpha_{\xi, \eta} \psi
$$

is an eigenfunction of $T_{\xi}$ with eigenvalue $\varepsilon+2$. Moreover,

$$
\|\hat{\psi}\|_{\xi}^{2}=\left(\varepsilon_{0}-\varepsilon\right)\|\psi\|_{\eta}^{2}
$$

Proof. By assumption $\psi \in \operatorname{Dom} T_{\eta}$. Hence, by Proposition 2.6. $\psi \in \operatorname{Dom} A_{\xi, \eta}$, and $\hat{\psi} \in H_{\xi}$. By (19) and Proposition 2.6, we have

$$
\begin{aligned}
\langle\hat{\psi}, \hat{\psi}\rangle_{\xi} & =-\left\langle B_{\eta, \xi} A_{\xi, \eta} \psi, \psi\right\rangle_{\eta} \\
& =\left\langle\left(\varepsilon_{0}-T_{\eta}\right) \psi, \psi\right\rangle_{\eta}=\left(\varepsilon_{0}-\varepsilon\right)\langle\psi, \psi\rangle_{\eta} .
\end{aligned}
$$

By construction,

$$
\begin{aligned}
\beta_{\eta, \xi} \hat{\psi} & =\beta_{\eta, \xi} \alpha_{\xi, \eta} \psi=\left(\varepsilon-\varepsilon_{0}\right) \psi \in \mathcal{H}_{\eta}, \\
\alpha_{\xi, \eta} \beta_{\eta, \xi} \hat{\psi} & =\left(\varepsilon-\varepsilon_{0}\right) \hat{\psi} \in \mathcal{H}_{\xi} .
\end{aligned}
$$

Hence, $\hat{\psi} \in \operatorname{Dom} B_{\eta, \xi}$ and $\beta_{\eta, \xi} \hat{\psi} \in \operatorname{Dom} A_{\xi, \eta}$. It follows that, $\hat{\psi} \in \operatorname{Dom} T_{\xi}$, with

$$
T_{\xi} \hat{\psi}=\left(A_{\xi, \eta} B_{\eta, \xi}+\varepsilon_{0}+2\right) \hat{\psi}=(\varepsilon+2) \hat{\psi} .
$$

Theorem 2.1. Let $\eta, \xi \in \mathcal{R}$ be such that (21) holds. Let $\sigma\left(T_{\eta}\right), \sigma\left(T_{\xi}\right)$ denote the spectral sets of the indicated operators. Then, $\varepsilon_{0}=\max \sigma\left(T_{\eta}\right)$. Moreover,

$$
\sigma\left(T_{\xi}-2\right)=\sigma\left(T_{\eta}\right) \backslash\left\{\varepsilon_{0}\right\} .
$$

Proof. Both $T_{\eta}$ and $T_{\xi}$ have a pure point spectrum; for a proof see Section 5.9 of [29] or Problem 1 in Ch. 9 of [2]. By assumption, $\psi_{0}:=\xi$ has no real zeros. Relation (21) is equivalent to

$$
\tau_{\eta} \psi_{0}=\varepsilon_{0} \psi_{0}
$$

Hence, $\varepsilon_{0}=\max \sigma\left(T_{\eta}\right)$ by the Sturm oscillation theorem. Let $\psi_{n} \in \mathcal{H}_{\eta}, n \in \mathbb{N}$ be the other eigenfunctions of $T_{\eta}$ with

$$
\tau_{\eta} \psi_{n}=\varepsilon_{n} \psi_{n}, \quad n=1,2, \ldots
$$

and $\varepsilon_{0}>\varepsilon_{1}>\varepsilon_{2}>\cdots$. Set

$$
\hat{\psi}_{n}=\alpha_{\xi, \eta} \psi_{n}, \quad n=1,2, \ldots
$$

By Proposition 2.7 these are all eigenfunctions of $T_{\xi}$ with eigenvalues $\varepsilon_{n}, n=$ $1,2, \ldots$. By Proposition 2.1, $\operatorname{Ran} A_{\xi, \eta}=\mathcal{H}_{\xi}$. Since $\left\{\psi_{n}\right\}_{n \in \mathbb{N}_{0}}$ is complete in $\mathcal{H}_{\eta}$, it follows that $\left\{\hat{\psi}_{n}\right\}_{n \in \mathbb{N}}$ is complete in $\mathcal{H}_{\xi}$. Therefore $\sigma\left(T_{\xi}\right)=\left\{\varepsilon_{n}\right\}_{n \in \mathbb{N}}$. 
Deift [3] proved a more general version of Theorem 2.1] regarding the spectra of general self-adjoint operators related by a factorization/Darboux transformation. Nonetheless, for the sake of comprehensiveness we prefer to state and prove a more restrictive version limited to Sturm-Liouville operators. The above proof of Theorem 2.1 is more accessible than Deift's more abstract argument.

\section{The formal theory of Exceptional Hermite polynomials}

Classical Hermite polynomials are orthogonal polynomials defined by the recurrence relation

$$
H_{0}=1, \quad x H_{n}=\frac{1}{2} H_{n+1}+n H_{n-1}, \quad n=1,2, \ldots
$$

They satisfy the following orthogonality relation:

$$
\int_{-\infty}^{\infty} H_{m}(x) H_{n}(x) e^{-x^{2}} d x=\sqrt{\pi} 2^{n} n ! \delta_{n, m}
$$

Hermite polynomials are known as classical orthogonal polynomials, because they also arise as solutions of the Hermite differential equation

$$
y^{\prime \prime}-2 x y^{\prime}=\varepsilon y
$$

with $y=H_{n}, \varepsilon=-2 n, n \in \mathbb{N}_{0}$. Note that multiplication of (32) by $-e^{-x^{2}}$ gives a singular Sturm-Liouville problem on $(-\infty, \infty)$, namely

$$
-\left(e^{-x^{2}} y\right)^{\prime}=-\varepsilon e^{-x^{2}} y \text {. }
$$

This SLP is limit-point at $\pm \infty$, so no explicit boundary conditions are required.

Exceptional Hermite polynomials are a generalization of the classical Hermite polynomials because they satisfy a second-order, Hermite-like differential equation. Each family of such polynomials is indexed by a partition, and so we begin by recalling some relevant definitions.

Definition 3.1. A partition $\lambda$ of a natural number $N$ is a non-increasing, finitely supported sequence of non-negative integers $\lambda_{1} \geq \lambda_{2} \geq \ldots \geq 0$ that sum to $N$. The length $\ell$ of a partition $\lambda$ is defined to be the smallest $\ell \in \mathbb{N}_{0}$ such that $\lambda_{\ell+1}=0$. Thus, $N=\lambda_{1}+\cdots+\lambda_{\ell}$, with $\lambda_{\ell}>0$.

Fix a partition $\lambda$, and let

$$
\begin{aligned}
C_{\lambda, l} & :=2^{l(l-1) / 2} \prod_{1 \leq i<j \leq l}\left(\lambda_{i}-\lambda_{j}+j-i\right) \\
\pi_{\lambda, l}(n) & :=\prod_{i=1}^{l}\left(n-N-\lambda_{i}+i\right), \quad k \in \mathbb{N}, \\
m_{i} & :=\lambda_{i}+\ell-i, \quad i \in \mathbb{N} ; \\
\eta_{\lambda} & :=\left(C_{\ell}^{\lambda}\right)^{-1} \operatorname{Wr}\left(H_{m_{\ell}}, \ldots, H_{m_{1}}\right), \\
I_{\lambda} & :=\left\{n \in \mathbb{N}_{0}: n-N+\ell \geq 0 \text { and } \pi_{\lambda, \ell}(n) \neq 0 .\right\}, \\
H_{\lambda, n} & :=\left(2^{\ell} C_{\ell}^{\lambda} \pi_{\lambda, \ell}(n)\right)^{-1} \operatorname{Wr}\left(H_{m_{\ell}}, \ldots, H_{m_{1}}, H_{n-N+\ell}\right), \quad n \in I_{\lambda}
\end{aligned}
$$

where Wr denotes the usual Wronskian determinant.

Observe that $n-N+\ell \in\left\{m_{1}, \ldots, m_{\ell}\right\}$ if and only if $\pi_{\lambda, \ell}(n)=0$. Thus, $H_{\lambda, n}, n \in I_{\lambda}$ is a non-zero Wronskian of classical Hermite polynomials. Also note 
that if $N=0$ and $\lambda$ is the empty partition, then $\eta_{\lambda}=1$ and $H_{\lambda, n}=H_{n}$, is the classical $n$th degree Hermite polynomial.

Proposition 3.1. Let $\eta_{\lambda}, H_{\lambda, n}, n \in I_{\lambda}$ be as above. Then

$$
\begin{aligned}
\eta_{\lambda}(x) & =2^{N} x^{N}+\text { lower degree terms. } \\
H_{\lambda, n}(x) & =2^{n} x^{n}+\text { lower degree terms. }
\end{aligned}
$$

Proof. Let $p_{i}, i=1, \ldots, l$ be a polynomial of degree $d_{i}$ with leading coefficient $c_{i}$. Suppose that $d_{1}, \ldots, d_{l}$ are distinct and let $P=\operatorname{Wr}\left(p_{1}, \ldots, p_{l}\right)$. Then

$$
\operatorname{deg} P=\sum_{i=1}^{l} d_{i}-\frac{1}{2} l(l-1)=\sum_{i=1}^{l}\left(d_{i}-l+i\right) .
$$

Consequently,

$$
\begin{aligned}
\operatorname{deg} \eta_{\lambda} & =\sum_{i=1}^{\ell}\left(m_{i}-\ell+i\right)=\sum_{i} \lambda_{i}=N, \\
\operatorname{deg} H_{\lambda, n} & =n-N+\ell-(\ell+1)+\ell+1+\sum_{i=1}^{\ell}\left(m_{i}-\ell-1+i\right)=n .
\end{aligned}
$$

The leading coefficient of $P$ is given by $\prod_{i=1}^{k} c_{i} \prod_{1 \leq i<j \leq k}\left(d_{j}-d_{i}\right)$. It follows that $C_{\lambda, \ell}$ and $2^{\ell} C_{\lambda, \ell} \pi_{\lambda, \ell}(n)$ are precisely the leading coefficients of $\eta_{\lambda}$ and $H_{\lambda, n}$, respectively.

The formulation of $\eta_{\lambda}$ and $H_{\lambda, n}$ in (36) and (38) is based on Wronskians of size $\ell$ and $\ell+1$ respectively. However, it is possible to define these polynomials using Wronskians of any size $l \geq \ell$.

Proposition 3.2. Let $\lambda$ be a partition of $N$ with length $\ell$. Fix $l \geq \ell$, and set

$$
m_{i, l}=\lambda_{i}+l-i, \quad i=1, \ldots, l .
$$

Then,

$$
\begin{aligned}
\eta_{\lambda} & =C_{\lambda, l}^{-1} \operatorname{Wr}\left(H_{m_{l, l}}, \ldots, H_{m_{1, l}}\right), \\
H_{\lambda, n} & =\left(2^{l} C_{l}^{\lambda} \pi_{\lambda, l}(n)\right)^{-1} \operatorname{Wr}\left(H_{m_{l, l}}, \ldots, H_{m_{l, 1}}, H_{n-N+l}\right)
\end{aligned}
$$

Proof. The proof is by induction on $l-\ell$. If $l=\ell$, there is nothing to prove. Suppose that (41) holds for a particular $l \geq \ell$. Observe that

$$
m_{i, l+1}=m_{i, l}+1, \quad i=1, \ldots, l,
$$

with $m_{l+1, l+1}=0$. Since

$$
H_{n}^{\prime}=2 n H_{n-1}, \quad n=1,2, \ldots,
$$

we have

$$
\begin{aligned}
\operatorname{Wr}\left(H_{m_{l+1, l+1}}, H_{m_{l+1, l}}, \ldots, H_{m_{l+1,1}}\right) & =\operatorname{Wr}\left(1, H_{m_{l, l}+1}, \ldots, H_{m_{l, 1}+1}\right) \\
& =\operatorname{Wr}\left(H_{m_{l, l}+1}^{\prime}, \ldots, H_{m_{l, 1}+1}^{\prime}\right) \\
& =2^{l} \prod_{i}\left(m_{l, i}+1\right) \operatorname{Wr}\left(H_{m_{l, l}}, \ldots, H_{m_{l, 1}}\right) .
\end{aligned}
$$


Observe that

$$
\sum_{i=1}^{l+1} m_{l+1, i}=N+l(l+1) / 2,
$$

the leading term coefficient of the left-side Wronskian is $2^{N} C_{\lambda, l+1}$, it follows that

$$
\operatorname{Wr}\left(H_{m_{l+1, l+1}}, H_{m_{l+1, l}}, \ldots, H_{m_{l+1,1}}\right)=C_{\lambda, l+1} \eta_{\lambda} .
$$

Relation (42) is proved in an anolgous fashion.

Corollary 3.1. For every $l \geq \ell$ and $n \in I_{\lambda}$, we have

$$
\frac{H_{\lambda, n}}{\eta_{\lambda}}=\frac{\operatorname{Wr}\left(H_{m_{l, l}}, \ldots, H_{m_{1, l}}, H_{n-N+l}\right)}{2^{l} \pi_{\lambda, l}(n) \operatorname{Wr}\left(H_{m_{l, l}}, \ldots, H_{m_{1, l}}\right)} .
$$

In other words, the rational function $\eta_{\lambda}^{-1} H_{\lambda, n}$ can be defined without invoking the normalizing constant $C_{\lambda, l}$ of (33).

A particularly interesting case of this formulation occurs when we take $l=N$. By (40), the set $I_{\lambda}$ is simply the degree sequence of the polynomial family $\left\{H_{\lambda, n}\right\}_{n}$. Let

$$
\begin{aligned}
k_{i} & :=m_{i, N}=\lambda_{i}+N-i, \quad i=1, \ldots, N, \\
K_{\lambda} & :=\left\{k_{1}, \ldots, k_{N}\right\}
\end{aligned}
$$

and observe that

$$
K_{\lambda}=\{0,1, \ldots, N-\ell-1\} \cup\left\{m_{i}+N-\ell: i=1, \ldots, \ell\right\} .
$$

Recall that $n-N+\ell \in\left\{m_{1}, \ldots, m_{\ell}\right\}$ if and only if $\pi_{\lambda, \ell}(n)=0$. It follows that $K_{\lambda}=\mathbb{N}_{0} \backslash I_{\lambda}$ is the set of exceptional degrees missing from $I_{\lambda}$, and that there are precisely $N=\operatorname{deg} \eta_{\lambda}$ such "exceptional" degrees. Moreover, by (41) and (42), we have

$$
\begin{aligned}
\eta_{\lambda} & \propto \operatorname{Wr}\left(H_{k_{N}}, \ldots, H_{k_{1}}\right), \\
H_{\lambda, n} & \propto \operatorname{Wr}\left(H_{k_{N}}, \ldots, H_{k_{1}}, n\right), \quad n \in I_{\lambda} .
\end{aligned}
$$

Thus both $\eta_{\lambda}$ and the exceptional polynomials $H_{\lambda, n}$ can be formulated as Wronskians involving the exceptional degrees. Of course, for most partitions $N>\ell$ and so the formulations in (36) and (38) are more economical. See 9] for a characterization of the smallest determinant that can be used to represent a Wronskian of Hermite polynomials.

Just like their classical counterparts, exceptional Hermite polynomials satisfy a second-order eigenvalue relation. Set

$$
\tau_{\lambda} y:=y^{\prime \prime}-2\left(x+\frac{\eta_{\lambda}^{\prime}}{\eta_{\lambda}}\right) y^{\prime}+\left(\frac{\eta_{\lambda}^{\prime \prime}}{\eta_{\lambda}}+2 x \frac{\eta_{\lambda}^{\prime}}{\eta_{\lambda}}\right) y
$$

i.e., $\tau_{\lambda}$ is the second-order differential expression in the left-side of (3) with $\eta=\eta_{\lambda}$.

Proposition 3.3. Let $m_{1}, \ldots, m_{l}, m \in \mathbb{N}_{0}$ be distinct non-zero integers. Set

$$
\begin{aligned}
& \eta=\operatorname{Wr}\left(H_{m_{l}}, \ldots, H_{m_{1}}\right) \\
& \xi=\operatorname{Wr}\left(H_{m_{l}}, \ldots, H_{m_{1}}, H_{m}\right)
\end{aligned}
$$

Then,

$$
\chi(\eta, \xi)=2(l-m) \eta \xi
$$


Proof. Relation (49) is equivalent to

$$
\left(\tau_{\eta}+2(m-l)\right) \xi=0
$$

Set

$$
\tilde{\tau}_{\eta} y=y^{\prime \prime}-2 x y^{\prime}+2(\log \eta)^{\prime \prime} y .
$$

An elementary calculation shows that

$$
\tau_{\eta}(\eta y)=\eta \tilde{\tau}_{\eta} y .
$$

Thus, it suffices to show that

$$
\left(\tilde{\tau}_{\eta}+2(m-l)\right)\left(\eta^{-1} \xi\right)=0 .
$$

Let

$$
\tau_{\emptyset} y=y^{\prime \prime}-2 x y^{\prime}
$$

be the classic Hermite differential expression. Define the $l$ th order differential expression

$$
\kappa y=\frac{\operatorname{Wr}\left(H_{m_{l}}, \ldots, H_{m_{1}}, y\right)}{\operatorname{Wr}\left(H_{m_{l}}, \ldots, H_{m_{1}}\right)}=y^{(l)}-(\log \eta)^{\prime} y^{(l-1)}+\cdots,
$$

and observe that

$$
\kappa H_{m}=\eta^{-1} \xi
$$

Since

$$
\tau_{\emptyset} H_{m}=-2 m H_{m},
$$

it suffices to show that

$$
\left(\tilde{\tau}_{\lambda}-2 l\right) \kappa=\kappa \tau_{\emptyset},
$$

This, in turn, is equivalent to the relation

$$
\left[\tau_{\emptyset}, \kappa\right]=\left(2 l-2(\log \eta)^{\prime \prime}\right) \kappa .
$$

By construction, $\operatorname{ker} \kappa=\operatorname{span}\left\{H_{m_{1}}, \ldots, H_{m_{l}}\right\}$. Since these are all eigenfunctions of $\tau_{\emptyset}$, the commutator $\left[\tau_{\emptyset}, \kappa\right]$ annihilates ker $\kappa$. By inspection, $\left[\tau_{\emptyset}, \kappa\right]$ has order $l$. Hence, $\left[\tau_{\emptyset}, \kappa\right]=f \kappa$ for some function $f(x)$. Observe that

$$
\left[\tau_{\emptyset}, \kappa\right] y=\left(2 l-2(\log \eta)^{\prime \prime}\right) y^{(l)}+\cdots
$$

Hence, $f=2 l-2(\log \eta)^{\prime \prime}$. This proves (51), which is equivalent to (49).

Corollary 3.2. Let $\lambda$ be a partition and $n \in I_{\lambda}$ an allowed degree. Then,

$$
\chi\left(\eta_{\lambda}, H_{\lambda, n}\right)=2(N-n) \eta_{\lambda} H_{\lambda, n} .
$$

Proof. Since $\chi$ is bilinear, this follows by (47) and the preceding Proposition.

The following converse was proved in $[8]$.

Proposition 3.4. Let $\eta(x)$ be a non-zero polynomial. Let $\left\{y_{n} \text { : } \operatorname{deg} y=n\right\}_{n \in I}$ be the family of polynomial solutions of

$$
\chi(\eta, y)=\varepsilon \eta y \quad \varepsilon \in \mathbb{C} .
$$

Suppose that $\mathbb{N}_{0} \backslash I$ is finite; i.e., the family of polynomial solutions is missing at most finitely many degrees. Then, there exists a partition $\lambda$ such that $I=I_{\lambda}$, and such that, up to a multiplicative constant, $\eta=\eta_{\lambda}$ and $y_{n}=H_{\lambda, n}, n \in I_{\lambda}$. 
3.1. Multi-step factorization chains. In this section, we show that every exceptional operator $T_{\lambda}$ is connected to the classical

$$
\tau_{\emptyset} y=y^{\prime \prime}-2 x y^{\prime}
$$

by a finite factorization chain of exceptional Hermite operators. We will describe two such chains: one will connect an exceptional Hermite operator $\tau_{\lambda}$ to $\tau_{\emptyset}$ and the other will connect $\tau_{\emptyset}$ to $\tau_{\lambda}$.

Let $\lambda$ be a partition of $N$. Let $k_{1}, \ldots, k_{N} \in K_{\lambda}$ be the exceptional degrees as per (44), and let $n_{1}, \ldots, n_{l} \in I_{\lambda}$ be a list of allowed degrees. Set

$$
\eta_{i}=\operatorname{Wr}\left(H_{k_{N}}, \ldots, H_{k_{1}}, H_{n_{1}}, \ldots, H_{n_{i}}\right), \quad i=0,1, \ldots, l,
$$

and introduce the differential expressions

$$
\begin{aligned}
\alpha_{i} & =\alpha_{\eta_{i+1}, \eta_{i}}, \quad i=0, \ldots, l-1, \\
\beta_{i} & =\alpha_{\eta_{i}, \eta_{i+1}} \\
\tau_{i} & =\tau_{\eta_{i}}, \quad i=0, \ldots, l .
\end{aligned}
$$

In particular, $\tau_{0}=\tau_{\lambda}$.

Proposition 3.5. With the above definitions, we have

$$
\begin{aligned}
\chi\left(\eta_{i}, \eta_{i+1}\right) & =2\left(N+i-n_{i+1}\right) \eta_{i} \eta_{i+1}, \quad i=0, \ldots, l-1, \\
\tau_{i} & =\beta_{i} \alpha_{i}+2\left(N+i-n_{i+1}\right), \quad i=0, \ldots, l-1 ; \\
\tau_{i+1} & =\alpha_{i} \beta_{i}+2\left(N+i+1-n_{i+1}\right) .
\end{aligned}
$$

Proof. Relation (53) follows by Corollary 3.2, Relations (54) and (55) follow by Lemma 2.2

Next, we describe a factorization chain that connects $\tau_{\lambda}$ to $\tau_{\emptyset}$ using $\lambda_{1}$ steps. Let $k_{1}, k_{2}, \ldots, k_{N} \in K_{\lambda}$ be the exceptional degrees, as per (44). Since $k_{1}=\lambda_{1}+N-1$, every $n \geq \lambda_{1}+N$ is an allowed degree; i.e. an element of $I_{\lambda}$.

Definition 3.2. We call the smallest $\lambda_{1}$ elements of $I_{\lambda}$ the sporadic degrees of $I_{\lambda}$.

Proposition 3.6. An $n \in I_{\lambda}$ is a sporadic degree if and only if there exists an exceptional degree $k \in K_{\lambda}$ such that $n<k$.

Proof. By (44), the $N$ exceptional degrees are contained in $\left\{0,1, \ldots, \lambda_{1}+N-1\right\}$, a set of cardinality $\lambda_{1}+N$. Hence, $n \in I_{\lambda}$ is dominated by some exceptional degree if and only if $n<\lambda_{1}+N-1$. By the preceding remark, there are precisely $\lambda_{1}$ such degrees.

Proposition 3.7. Let $\lambda$ be a partition of $N$, and let $n_{1}<n_{2}<\ldots$ be the allowed degrees in $I_{\lambda}$ listed in increasing order. For $j \in \mathbb{N}_{0}$, set

$$
\lambda_{i}^{(j)}=\max \left(\lambda_{i}-j, 0\right) \quad i \in \mathbb{N},
$$

and let $\ell_{j}$ be the length of $\lambda^{(j)}$. Then,

$$
\ell_{j}=N-n_{j+1}+j, \quad j \in \mathbb{N}_{0} .
$$

Proof. Observe that if $j \geq \lambda_{1}$, then $\lambda^{(j)}=\emptyset$ is the trivial partition. Thus it suffices to establish (57) for $j=0, \ldots, \lambda_{1}-1$. 
By definition, $i \leq \ell_{j}$ if and only if $\lambda_{i}-j \geq 1$. In other words, the sequence $\left(\ell_{0}, \ell_{1}, \ell_{2}, \ldots\right)$ and $\lambda$ are dual partitions. Observe that if $\lambda_{i}-j \geq 1$, then $i-\ell_{j} \leq 0$. Conversely, $\lambda_{i}-j \leq 0$ if and only if $i-\ell_{j} \geq 1$. Hence,

$$
\lambda_{i}-i \neq j-\ell_{j}, \quad i \in \mathbb{N}, j \in \mathbb{N}_{0} .
$$

Using $\sqcup$ for disjoint union, it follows that

$$
\left\{\lambda_{i}-i: i=1, \ldots, N\right\} \sqcup\left\{j-\ell_{j}: j=0, \ldots, \lambda_{1}-1\right\}=\left\{-N,-N+1, \ldots, \lambda_{1}-1\right\} .
$$

Therefore,

$$
\left\{j-\ell_{j}+N: j=0, \ldots, \lambda_{1}-1\right\}
$$

is precisely the set of sporadic degrees in $I_{\lambda}$. Relation (57) follows.

Proposition 3.8. Let $\lambda$ be a partition, with $\lambda^{(j)}, j \in \mathbb{N}_{0}$ the sequence of partitions defined by (56). Define the differential expressions

$$
\begin{aligned}
\alpha_{j} & =\alpha_{\eta_{\lambda}(j+1)}, \eta_{\lambda(j)}, \quad j=0, \ldots, \lambda_{1}-1, \\
\beta_{j} & =\alpha_{\eta_{\lambda}(j)}, \eta_{\lambda}(j+1) \\
\tau_{j} & =\tau_{\eta_{\lambda}(j)}, \quad j=0, \ldots, \lambda_{1} .
\end{aligned}
$$

Then,

$$
\begin{aligned}
\tau_{j} & =\beta_{j} \alpha_{j}+2 \ell_{j}, \quad j=0, \ldots, \lambda_{1}-1 ; \\
\tau_{j+1} & =\alpha_{j} \beta_{j}+2\left(\ell_{j}+1\right) .
\end{aligned}
$$

Proof. Let $n_{1}<\ldots<n_{\lambda_{1}}$ be the sporadic degrees of $\lambda$ listed in increasing order. Let

$$
\begin{aligned}
K_{j} & =K_{\lambda} \cup\left\{n_{1}, \ldots, n_{j}\right\}, \quad j=0, \ldots, \lambda_{1}, \\
\eta_{j} & =\operatorname{Wr}\left(k_{N}, \ldots, k_{1}, n_{1}, \ldots, n_{j}\right) .
\end{aligned}
$$

By (57) and (53),

$$
\chi\left(\eta_{j}, \eta_{j+1}\right)=2\left(N+j-n_{j+1}\right) \eta_{j} \eta_{j+1}=2 \ell_{j} \eta_{j} \eta_{j+1} .
$$

The desired conclusion now follows by Proposition 3.5 .

For example, consider the partition $\lambda=(3,3,1,1,0, \ldots)$ of $N=8$. The exceptional degrees are

$$
K_{\lambda}=\{0,1,2,3,5,6,9,10\} .
$$

The degree set is therefore,

$$
I_{\lambda}=\{4,7,8,11,12,13, \ldots\},
$$

with $4,7,8$ being the sporadic degrees. In terms of the above terminology,

$$
\begin{array}{lll}
K_{1}=\{0,1,2,3,4,5,6,9,10\}, & \lambda^{(1)}=(2,2,0,0,0, \ldots), & N_{1}=4, \ell_{1}=2 \\
K_{2}=\{0,1,2,3,4,5,6,7,9,10\}, & \lambda^{(2)}=(1,1,0,0,0, \ldots), & N_{2}=2, \ell_{2}=2 \\
K_{3}=\{0,1,2,3,4,5,6,7,8,9,10\}, & \lambda^{(3)}=(0,0,0,0,0, \ldots), & N_{3}=0, \ell_{3}=0 .
\end{array}
$$


3.2. The norm identity. In this section, we present and prove a certain algebraic identity that will allow us to derive a formula for the norming constants of the exceptional Hermite polynomials.

Proposition 3.9. Let $m_{1}, \ldots, m_{l}, m \in \mathbb{N}_{0}$ be distinct non-negative integers. Set

$$
\begin{aligned}
\eta_{0} & =1 \\
\xi_{0} & =H_{m} \\
\eta_{i} & =\operatorname{Wr}\left(H_{m_{1}}, \ldots, H_{m_{i}}\right), \quad i=1, \ldots, l ; \\
\xi_{i} & =\operatorname{Wr}\left(H_{m_{1}}, \ldots, H_{m_{i}}, H_{m}\right)
\end{aligned}
$$

Also, let $\rho_{0}=0$, and recursively define

$$
\rho_{i+1}=\frac{\xi_{i} \xi_{i+1}}{\eta_{i} \eta_{i+1}}+2\left(m-m_{i+1}\right) \rho_{i}, \quad i=0, \ldots, l-1
$$

We then have,

$$
\left(\frac{\xi_{l}}{\eta_{l}}\right)^{2} e^{-x^{2}}-2^{l} \prod_{i=1}^{l}\left(m-m_{i}\right) \xi_{0}^{2} e^{-x^{2}}=\left(\rho_{l} e^{-x^{2}}\right)^{\prime}
$$

Proof. By (49), we have

$$
\chi\left(\eta_{i}, \eta_{i+1}\right)=2\left(i-m_{i+1}\right) \eta_{i} \eta_{i+1}, \quad i=0, \ldots, l-1 .
$$

Hence, by the argument used in Proposition 3.5, the differential expressions

$$
\begin{aligned}
\alpha_{i} & =\alpha_{\eta_{i+1}, \eta_{i}}, \quad i=0, \ldots, l-1 \\
\beta_{i} & =\beta_{\eta_{i}, \eta_{i+1}}, \quad i=0, \ldots, l-1 \\
\tau_{i} & =\tau_{\eta_{i}}, \quad i=0, \ldots, l .
\end{aligned}
$$

constitute the factorization chain

$$
\begin{aligned}
\tau_{i} & =\beta_{i} \alpha_{i}+2\left(i-m_{i+1}\right), \quad i=0, \ldots, l-1 \\
\tau_{i+1} & =\alpha_{i} \beta_{i}+2\left(i+1-m_{i+1}\right) .
\end{aligned}
$$

Next, observe that

$$
\begin{aligned}
\alpha_{i} \xi_{i} & =\frac{\operatorname{Wr}\left(\operatorname{Wr}\left(H_{m_{1}}, \ldots, H_{m_{i}}, H_{m_{i+1}}\right), \operatorname{Wr}\left(H_{m_{1}}, \ldots, H_{m_{i}}, H_{m}\right)\right)}{\operatorname{Wr}\left(H_{m_{1}}, \ldots, H_{m_{i}}\right)} \\
& =\operatorname{Wr}\left(H_{m_{1}}, \ldots, H_{m_{i}}, H_{m_{i+1}}, H_{m}\right)=\xi_{i+1} .
\end{aligned}
$$

Again, by (49), we have

$$
\tau_{i}\left(\xi_{i}\right)=2(i-m) \xi_{i}, \quad i=0, \ldots, l .
$$

Hence, by (13), we have

$$
\frac{\xi_{i+1}^{2}}{\eta_{i+1}^{2}} e^{-x^{2}}-2\left(m-m_{i+1}\right) \frac{\xi_{i}^{2}}{\eta_{i}^{2}} e^{-x^{2}}=\left(\frac{\xi_{i} \xi_{i+1}}{\eta_{i} \eta_{i+1}} e^{-x^{2}}\right)^{\prime}, \quad i=0, \ldots, l-1 .
$$

Hence, by induction,

$$
\frac{\xi_{l}^{2}}{\eta_{l}^{2}} e^{-x^{2}}-2^{l} \prod_{i=1}^{l}\left(m-m_{i}\right) \xi_{0}^{2} e^{-x^{2}}=\left(e^{-x^{2}} \sum_{i=1}^{l} \prod_{j=1+i}^{l}\left(2 m-2 m_{j}\right) \frac{\xi_{j} \xi_{j-1}}{\eta_{j} \eta_{j-1}}\right)^{\prime}
$$


Again, by induction,

$$
\rho_{k}=\sum_{i=1}^{k} \prod_{j=1+i}^{l}\left(2 m-2 m_{j}\right) \frac{\xi_{j} \xi_{j-1}}{\eta_{j} \eta_{j-1}}, \quad k=1, \ldots, \ell-1 .
$$

The desired identity (60) follows immediately.

\section{THE $\mathrm{L}^{2}$ THEORY}

The following theorem was proved by Krein for Sturm-Liouville problems on the half-line, and independently by Adler for Sturm-Liouville problems on a bounded interval. The Adler argument can be extended without difficulty to the case of an infinite interval [7].

Definition 4.1. We say that $\lambda$ is an even partition $\lambda_{2 i-1}=\lambda_{2 i}$ for every $i \in \mathbb{N}$.

Theorem 4.1 (Krein-Adler). The polynomial $\eta_{\lambda}(x)$ has no real zeros if and only if $\lambda$ is an even partition.

Henceforth we assume that $\lambda$ is an even partition. In that case,

$$
W_{\lambda}:=W_{\eta_{\lambda}}=\eta_{\lambda}^{-2} e^{-x^{2}}
$$

is a non-singular weight on $(-\infty, \infty)$ with finite moments. Let $\mathcal{H}_{\lambda}=\mathrm{L}^{2}\left(\mathbb{R}, W_{\lambda}\right)$ denote the corresponding Hilbert space with weighted inner product,

$$
\langle f, g\rangle_{\lambda}=\int_{\mathbb{R}} f \bar{g} W_{\lambda}=\int_{R} \frac{f}{\eta_{\lambda}} \frac{\bar{g}}{\eta_{\lambda}} e^{-x^{2}}, \quad f, g \in \mathcal{H}_{\lambda} .
$$

Proposition 4.1. Let $\lambda$ be an even partition. Then, the corresponding exceptional Hermite polynomials enjoy the following orthogonality property:

$$
\left\langle H_{\lambda, n}, H_{\lambda, n^{\prime}}\right\rangle_{\lambda}=\sqrt{\pi} 2^{n-N} \frac{n !}{\pi_{\lambda, N}(n)} \delta_{n, n^{\prime}}, \quad n, n^{\prime} \in I_{\lambda},
$$

with $\pi_{\lambda, N}(n)$ as defined in (34).

Proof. Being the eigenfunctions of a SLP (4), the $H_{\lambda, n}, n \in I_{\lambda}$ are orthogonal with respect to $W_{\lambda}=W_{\eta_{\lambda}}$. It remains to establish the form of the norming constants in (61). By Corollary 3.1 and (600),

$$
\begin{aligned}
\left\langle H_{\lambda, n}, H_{\lambda, n}\right\rangle_{\lambda} & =\int_{\mathbb{R}}\left(\frac{H_{\lambda, n}(x)}{\eta_{\lambda}(x)}\right)^{2} e^{-x^{2}} d x, \quad n \in I_{\lambda} \\
& =\int_{\mathbb{R}}\left(\frac{\operatorname{Wr}\left(H_{k_{N}}, \ldots, H_{k_{1}}, H_{n}\right)}{2^{N} \pi_{\lambda, N}(n) \operatorname{Wr}\left(H_{k_{N}}, \ldots, H_{k_{1}}\right)}\right)^{2} e^{-x^{2}} d x \\
& =\frac{1}{2^{N} \pi_{\lambda, N}(n)} \int_{R} H_{n}(x)^{2} e^{-x^{2}} d x \\
& =\sqrt{\pi} 2^{n-N} \frac{n !}{2^{N} \pi_{\lambda, N}(n)} .
\end{aligned}
$$

Theorem 4.2. Let $\lambda$ be an even partition. Then the SLP of form (5) with $\eta=\eta_{\lambda}$ has eigenvalues

$$
\varepsilon_{n}=2(n-N), \quad n \in I_{\lambda}
$$

The corresponding eigenfunctions are $H_{\lambda, n}, n \in I_{\lambda}$, while the norming constants are given by (61). 
Lemma 4.1. Let $\lambda$ be a partition and let $\lambda^{(j)}, j \in \mathbb{N}$ be the partitions defined in (56). If $\lambda$ is an even partition, then so is every $\lambda^{(j)}$.

Proof. Suppose that $\lambda_{2 i-1}=\lambda_{2 i}$ for all $i \in \mathbb{N}$. It follows that

$$
\lambda_{2 i-1}^{(j)}=\max \left(\lambda_{2 i-1}-j, 0\right)=\max \left(\lambda_{2 i}-j, 0\right)=\lambda_{2 i}^{(j)} \quad i, j \in \mathbb{N} .
$$

of Theorem 4.2. Multiplication of (4) by $-W_{\lambda}^{-1}$ and changing $\varepsilon \rightarrow-\varepsilon$, gives (3), which may be expressed as

$$
\tau_{\lambda} y=\varepsilon y
$$

By (12) and (52), we have

$$
\tau_{\lambda} H_{\lambda, n}=2(N-n) H_{\lambda, n}, \quad n \in I_{\lambda} .
$$

Hence, $2(n-N)$ is an eigenvalue of (5) with corresponding eigenfunction $H_{\lambda, n}, n \in$ $I_{\lambda}$.

It remains to show that there are no either eigenvalues; i.e., that $\left\{H_{\lambda, n}\right\}_{n \in I_{\lambda}}$ is a complete orthogonal basis. To show this, we employ the factorization chain described in Proposition 3.8. Let $\lambda^{(j)}, j=0, \ldots, \lambda_{1}$ be the partitions defined in (56), and let $\eta_{j}=\eta_{\lambda^{(j)}}$. By the above Lemma, these are all even partitions, and hence each $\eta_{j} \in \mathcal{R}$.

Let $T_{j}=T_{\eta_{j}}, j=0,1, \ldots, \lambda_{1}$ be a sequence of self-adjoint operators with action $\tau_{\eta_{j}}$ and domains as per (20). By Proposition 2.6. the formal factorization chain of Proposition 3.8 corresponds to a factorization chain of operators

$$
\begin{aligned}
T_{j} & =B_{j} A_{j}+2 \ell_{j}, \quad j=0, \ldots, \lambda_{1}-1, \\
T_{j+1} & =A_{j} B_{j}+2 \ell_{j}+2, \quad j=0, \ldots, \lambda_{1}-1,
\end{aligned}
$$

where $\ell_{j}$ is the length of partition $\lambda^{(j)}$, and where $A_{j}, B_{j}$ are first order operators with action $\alpha_{\eta_{j+1}, \eta_{j}}, \beta_{\eta_{j+1}, \eta_{j}}$, respectively, and domains as given in (18). Hence, $2 \ell_{j}$ is the largest eigenvalue of $T_{j}, j=0, \ldots, \lambda_{1}$. Hence, by Theorem 2.1,

$$
\sigma\left(T_{j+1}-2\right)=\sigma\left(T_{j}\right) \backslash\left\{2 \ell_{j}\right\} .
$$

It follows that

$$
\sigma\left(T_{0}\right)=\left\{2 \ell_{j}-2 j: j=0, \ldots, \lambda_{1}-1\right\} \cup \sigma\left(T_{\lambda_{1}}\right) .
$$

However, since $\tau_{\lambda_{1}}=\tau_{\emptyset}$ is the classical Hermite operator, we have by (57) that

$$
\begin{aligned}
\sigma\left(T_{0}\right) & =\left\{2 \ell_{j}-2 j: j=0, \ldots, \lambda_{1}-1\right\} \cup\left(-2 \mathbb{N}_{0}\right) \\
& =\left\{2\left(N-n_{j+1}\right): j=0, \ldots, \lambda_{1}-1\right\} \cup\left(-2 \mathbb{N}_{0}\right) \\
& =\left\{2(N-n): n \in I_{\lambda}\right\} .
\end{aligned}
$$

Corollary 4.1. Let $K=\left\{k_{1}, \ldots, k_{N}\right\} \subset \mathbb{N}_{0}$ be a list of $N$ non-negative integers such that

$$
\sum_{i=1}^{N} k_{i}=\frac{1}{2} N(N+1)
$$

Set

$$
\lambda_{i}=k_{i}+i-N, i \in \mathbb{N} .
$$


Then, $\left\{H_{\lambda, n}\right\}_{n \in I_{\lambda}}$ is a family of exceptional Hermite polynomials such that $K=$ $K_{\lambda}$; i.e. the exceptional degrees are precisely $K$. Moreover, if $\lambda$ is an even partition, then the corresponding $\left\{H_{\lambda, n}\right\}_{n \in I_{\lambda}}$ are eigenfunctions of the SLP (4) whose spectrum, up to a shift with $N$, differs from the spectrum of the classical Hermite $S L P$ by the removal of eigenvalues at position $2 k, k \in K$.

\section{REFERENCES}

[1] Bochner, S.: On Sturm-Liouville Polynomial Systems, Math. J. 29 730-736 (1929)

[2] Coddington, E.A. and Levinson, N.: Theory of ordinary differential equations. Tata McGrawHill Education, (1955)

[3] Deift, P.A.: Applications of a commutation formula, Duke Math. J. 45, 267-310 (1978)

[4] Dubov, S.Y., Eleonskii, V.M. and Kulagin, N. E.: Equidistant spectra of anharmonic oscillators., Chaos 4, 47-53 (1994).

[5] Durán, A. J.: Higher order recurrence relation for exceptional Charlier, Meixner, Hermite and Laguerre orthogonal polynomials, Integr. Transf. Spec. F. 26, 357-376 (2015)

[6] García-Ferrero, M. A., Gómez-Ullate, D. and Robert Milson: A Bochner type characterization theorem for exceptional orthogonal polynomials, J. Math. Anal. Appl. 472, 584-626 (2019)

[7] García-Ferrero, M.A. and Gómez-Ullate, D.: Oscillation theorems for the Wronskian of an arbitrary sequence of eigenfunctions of Schrödinger's equation. Lett. Math. Phys. 105, 551$573(2015)$

[8] Gomez-Ullate, D., Grandati, Y. and Milson, R.: Rational extensions of the quantum harmonic oscillator and exceptional Hermite polynomials. Journal of Physics A: Mathematical and Theoretical 47, 015203 (2014)

[9] Gomez-Ullate, D., Grandati, Y. and Milson, R.: Durfee rectangles and pseudo-Wronskian equivalences for Hermite polynomials, Stud. Appl. Math. 141, 596-625, (2018)

[10] Gomez-Ullate, D., Kamran, N. and Milson, R.: An extended class of orthogonal polynomials defined by a Sturm-Liouville problem, J. Math. Anal. Appl. 359, 352-367 (2009)

[11] Gomez-Ullate, D., Kamran, N. and Milson, R.: Exceptional orthogonal polynomials and the Darboux transformation, J. Phys A 43, 434016 (2010)

[12] Gomez-Ullate, D., Kamran, N. and Milson, R.: Two-step Darboux transformations and exceptional Laguerre polynomials, J. Math. Anal. and Appl. 387, 410-418 (2012)

[13] Gómez-Ullate, D., Kasman, A., Kuijlaars, A. B. J. and Milson, R.: Recurrence Relations for Exceptional Hermite Polynomials, J. Approx. Theory 204, 1-16 (2016)

[14] Gómez-Ullate, D., Marcellán, F. and Milson, R.: Asymptotic and interlacing properties of zeros of exceptional Jacobi and Laguerre polynomials, J. Math. Anal. Appl. 399, 480-495 (2013)

[15] Gesztesy, F., and Teschl, G: On the double commutation method. Proc. American Math. Soc. 124, 1831-1840 (1996)

[16] Hemery, A. D. and Veselov, A. P. : Whittaker-Hill equation and semifinite-gap Schrödinger operators, J. Math. Phys. 51, 072108 (2010)

[17] Horváth, Á. P.: The electrostatic properties of zeros of exceptional Laguerre and Jacobi polynomials and stable interpolation, J. Approx. Theory 194, 87-107, (2015)

[18] Kuijlaars, A.B.J. and Milson, R.: Zeros of exceptional Hermite polynomials, J. Approx. Theory 200, 28-39, (2015)

[19] Kasman, A. and Milson, R.: The Adelic Grassmannian and Exceptional Hermite Polynomials, Mathematical Physics, Analysis and Geometry 23, 1-51 (2020)

[20] Liaw, C., Littlejohn, L and Kelly J.S.: Spectral analysis for the exceptional $X_{m}$-Jacobi equation. Electronic Journal of Differential Equations 194, 1-10 (2015)

[21] Liaw, C., Littlejohn, L., Kelly J.S. and Milson, R.: The spectral analysis of three families of exceptional Laguerre polynomials. Journal of Approximation Theory 202, 5-41 (2016)

[22] Marquette, M. and Quesne, C.: New families of superintegrable systems from Hermite and Laguerre exceptional orthogonal polynomials, J. Math. Phys. 54042102 (2013)

[23] Odake, S. and Sasaki, R: Infinitely many shape invariant potentials and new orthogonal polynomials, Phys. Lett. B 679, 414-417 (2009)

[24] Post, S. Tsujimoto, S. and Vinet, L.: Families of superintegrable Hamiltonians constructed from exceptional polynomials, J. Phys. A 45, 405202 (2012) 
[25] Quesne, C.: Solvable rational potentials and exceptional orthogonal polynomials in supersymmetric quantum mechanics, SIGMA 5, (2009)

[26] Schulze-Halberg, A. and Roy, B.: Darboux partners of pseudoscalar Dirac potentials associated with exceptional orthogonal polynomials, Ann. Phys. 349, 159-170 (2014)

[27] Rudin, W.: Functional analysis. McGraw-Hill, New York (1991)

[28] Sasaki, R., Tsujimoto, S. and Zhedanov, A.: Exceptional Laguerre and Jacobi polynomials and the corresponding potentials through Darboux-Crum transformations, J. Phys. A 43 315204 (2010)

[29] E.C. Titchmarsh: Eigenfunction Expansions Associated with Second-order Differential Equations Part 1, Oxford University Press, London (1962)

Escuela Superior de Ingeniería, U. Cádiz, 11519 Puerto Real, Spain, and Departamento de Física Teórica, U. Complutense, 28040 Madrid, Spain

Email address: david.gomezullate@uca.es

L. Physique et Chimie Théoriques, U. de Lorraine, 57078 Metz, Cedex 3, France

Email address: yves.grandati@univ-lorraine.fr

Dept. of Mathematics and Statistics, Dalhousie U., Halifax NS, Canada B3H 3J5

Email address: rmilson@dal.ca 